\title{
Uric acid - history, present and future
}

\begin{tabular}{|c|}
\hline 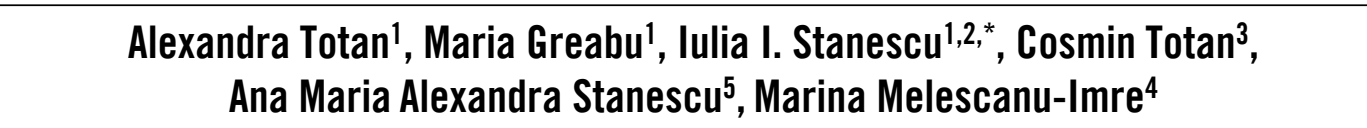 \\
\hline $\begin{array}{l}\text { 1Department of Biochemistry, Faculty of Dental Medicine, } \\
\text { "Carol Davila" University of Medicine and Pharmacy, Bucharest } \\
\text { 2Department of Physiology, Faculty of Dental Medicine, } \\
\text { "Carol Davila" University of Medicine and Pharmacy, Bucharest } \\
\text { 3Department of Oral and Maxillofacial Surgery, Faculty of Dental Medicine, } \\
\text { "Carol Davila” University of Medicine and Pharmacy, Bucharest } \\
\text { "Department of Complete Denture, Faculty of Dental Medicine, } \\
\text { "Carol Davila" University of Medicine and Pharmacy, Bucharest } \\
{ }^{5} \text { Department of Family Medicine, „Carol Davila" University of Medicine and Pharmacy, Bucharest }\end{array}$ \\
\hline
\end{tabular}

\begin{abstract}
2,6,8-trioxypurine known as uric acid represents a heterocyclic derivative of purine, with a molecular weight of $158 \mathrm{Da}$. In contrast to primates and humans, in the other animals uric acid is further oxidized by the uricase enzyme (urate oxidase) to allantoin, 100 times more water-soluble than uric acid and, consequently, more efficient excreted in the urine. Humans and higher pri-mates lack a functional uricase gene. The uricase activity loss in higher primates and humans occurred about 15 million years ago, re $\urcorner$ sulting in a relatively higher plasma uric acid level than that in lower ani-mals. This was an impressive and very important evolutionary event, providing essential survival advantages for humans. Uric acid represents a powerful antioxidant capable of scavenging singlet oxygen molecules, oxygen radicals, and peroxynitrite (ONOO-) molecules. However, in vivo and cellular research studies are interestingly illustrating that, depending on its chemical microenvironment, the uric acid molecule plays a duplicate role, being also a pro-oxidant. Uric acid represents the most important salivary antioxidant, in the circumstances of very low salivary thiols concentrations. Uric acid plays an important role in oral pathology and may be regarded as a very useful future salivary biomarker.
\end{abstract}

Keywords: uric acid, oxidative stress, antioxidant

\section{URIC ACID'S HISTORY}

2,6,8-trioxypurine known as uric acid represents a heterocyclic derivative of purine, with a molecular weight of $158 \mathrm{Da}$.

Uric acid was first identified as a bladder stones component by the Swedish pharmacist Scheele, in $1776(1)$.

In the terms of acid-base uric acid is a weak acid which forms a singly-charged hydrogen (acid urate ion) at biological $\mathrm{pH}$ (1). Uric acid's water solubility is relatively low and depends on the environment temperature. At $37^{\circ} \mathrm{C}$ and $\mathrm{pH} 7.4$ the uric acid saturation point is $392 \mu \mathrm{mol} / 1(6.6 \mathrm{mg} / \mathrm{dl})(1)$.

Remarkably, in higher primates and humans, uric acid represents the final breakdown product of purine (adenine and guanine) nucleotides metabo- lism (1). Purine nucleotides represent the main constituents of nucleic acids and cellular energy stores (ATP, GTP) (2).

In the kidney, at least $90 \%$ of the filtered uric acid is reabsorbed by the URAT 1 and GLUT9 specific anion transporters (1).URAT 1 anion transporter reabsorbs urate and secretes instead anionic organic molecules (lactate, ketone bodies or xenobiotics) through a counter-transport process (1).

In contrast to primates and humans, in the other animals uric acid is further oxidized by the uricase enzyme (urate oxidase) to allantoin, 100 times more water-soluble than uric acid and, consequently, more efficient excreted in the urine (1). Humans and higher primates lack a functional uricase gene (1).

The metabolic pathway of uric acid generation briefly reviews the following steps (2): 
- inosine monophosphate (IMP) is formed from de novo purine synthesis. De novo purine nucleotide synthesis leads to IMP via ribose-5-phosphate

- IMP is also formed and during purine salvage pathway. In the salvage pathway, hypoxanthine-guanine phosphoribosyl transferase (HGPRT) plays an important role in IMP generation

- IMP is then metabolized to hypoxanthine

- in the next step hypoxanthine is oxidased to xanthine by xanthine oxidase

- finally, xanthine is oxidased to uric acid, also by xanthine oxidase $(\mathrm{XO})$

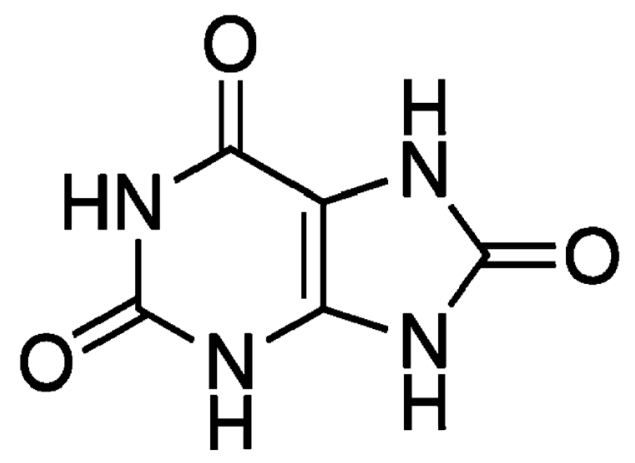

FIGURE 1. Uric acid molecule

Normally in humans, the plasma uric acid levels are (1):

- 2.6-5.7 mg/dl (155-339 $\mu \mathrm{mol} / \mathrm{l})$ for premenopausal women

- $3.5-7.0 \mathrm{mg} / \mathrm{dl}(208-416 \mu \mathrm{mol} / \mathrm{l})$ for men and postmenopausal women

In most of the other mammals normal plasma uric acid levels are 1-2 mg/dl due to the further oxidation reaction to allantoin by uricase $(1,3)$.

The ancient egyptians first described the gout as a disease (4). Hippocrates, however, defined gout as "arthritis of the rich" distinguishing it from "arthritis of the poor" (4).

In 1683, The English physician Thomas Sydenham was the first who described the association between hyperuricemia, gout, and high standard of living (4). He, also, supposed that hyperuricemia was related to good nutrition, and to greater intelligence and creativity ("gout kills more wise men than simple") (4).

\section{HOW SHOULD URIC ACID BE REGARDED IN PRESENT DAYS}

The uricase activity loss in higher primates and humans occurred about 15 million years ago, re- sulting in a relatively higher plasma uric acid level than that in lower animals. This was an impressive and very important evolutionary event, providing essential survival advantages for humans (1).

These evolutionary advantages are the increased blood antioxidant capacity and the ability to adjust sodium retention and, consequently, the blood pressure (1). Higher plasma uric acid levels should be also linked (via increasing the antioxidant capacity) to intelligence fulminant evolution, longevity and innate immune functions improvement. During primates evolution the loss of ascorbate synthetase was followed by the loss of uricase. Most probably, blood uric acid replaced or supplemented the antioxidant functions of ascorbate during primates evolution $(3,5)$.

Uric acid represents a powerful antioxidant capable of scavenging singlet oxygen molecules, oxygen radicals, and peroxynitrite (ONOO-) molecules. Uric acid molecule also can chelates transition metals in order to reduce ion-mediated ascorbic acid oxidation (5). Duet o its chemical structure, uric acid is responsible for approximately $50 \%$ of serum antioxidant activity (5). The uric acid molecule can be regarded as an exceptional peroxynitrite $\left(\mathrm{ONOO}^{-}\right)$scavenger in the extracellular fluid (6). However, it is important to highlight that the uric acid cannot scavenge the superoxide anion (6). Moreover, as an efficient antioxidant molecule, uric acid needs the presence of ascorbic acid and thiols, in order to complete peroxynitrites scavenging (6). Interestingly, ascorbate by itself is not able to prevent the reaction between peroxynitrite and tetrahydrobiopterin. This illustrates the major role of uric acid on the peroxynitrite scavenging scene (5).

However, in vivo and cellular research studies are interestingly illustrating that, depending on its chemical microenvironment, the uric acid molecule plays a duplicate role, being also a pro-oxidant (7). Strong epidemiological evidence suggests that the prevalence of gout and hyperuricemia is increasing worldwide (8). Plasma uric acid levels higher than $7 \mathrm{mg} / \mathrm{dl}$ are significantly associated with visceral obesity, insulin resistance, dyslipidemia, type II diabetes, kidney disease, hypertension and cardiovascular events $(9,10)$.

Despite the evolutionary advantages, in the modern era the high plasma uric acid levels may become serious risk factors for many human diseases most of which being included into the metabolic syndrome category (4). The modern life led to increased consumption of purine-rich foods, alcohol, and soft drinks sweetened with fructose, which 
triggered the uric acid overproduction and higher plasma levels, termed hyperuricemia (1). Also, hyperuricemia could be induced by the under-excretion of uric acid from the kidney as a result of renal dysfunction or influence of certain metabolites and medications (1).

So, the uric acid metabolism should be regarded as „,double-edged sword” (4). As long as, concerning the inflammatory and/or oxidative responses, in many organs, its benefits are overcome by harmful effects, leading to the most leading to the most common metabolic diseases of our days.

The higher plasma uric acid level, selected during primates evolution, is regarded as beneficial in the context of oxidative stress (1). Antioxidant capacity of uric acid consists in neutralizing prooxidant molecules, such as hydroxyl radicals, hydrogen peroxide, and peroxynitrite via nonenzymatic oxidation (5). As a result of uric acid antioxidant action, allantoin is a major end product of urate oxidation in the peroxide/heme system (5). Due to the fact that humans lack uricase (the enzyme which converts urate in allantoin), allantoin urine, blood and especially, saliva, should be of considerable interest as an indicator of urate oxidation, illustrating the oxidative stress level (5).

\section{REFERENCES}

1. Chen C, Lu JM., Yao Q. Hyperuricemia-Related Diseases and Xanthine Oxidoreductase (XOR) Inhibitors: An Overview. Med Sci Monit, 2016; 22: 2501-2512

2. Greabu M, Totan A. Tratat de chimie şi biochimie pentru Medicina Dentară, Vol I si II. Editura Standardizarea, Bucureşti , 2010.

3. Greabu M, Battino M, Totan A, Mohora M, Mitrea N, Totan C, Spinu T, Didilescu A. Effect of gas phase and particulate phase of cigarette smoke on salivary antioxidants. What can be the role of vitamin $C$ and pyridoxine? Pharmacol Rep. 2007 Sep-Oct;59(5):613-8.

4. De Giorgi A, Fabbian F, Pala M, Tiseo R, Parisi C, Misurati E, Manfredini R. Uric acid: friend or foe? Uric acid and cognitive function "Gout kills more wise men than simple". European Review for Medical and Pharmacological Sciences 2015; 19: 640-646

5. Totan A, Balcangiu-Stroescu AE, Imre M, Miricescu M, Balan D, Stanescu II, Ionescu D, Timofte D, Tanasescu MD, Greabu M. XOR Possible Correlations with Oxidative Stress and Inflammation Markers in the Context of Diabetic Kidney Disease. Revista de chimie, 2019, 70(4), 1396-98

6. Yao JK, Dougherty GG, Reddy RD, MatsonWR, Rima KaddurahDaouk R, Matcheri S Keshavan MS. Associations between purine metabolites and monoamine neurotransmitters in first-episode psychosis. Frontiers in Cellular Neuroscience, 2013, 7.

\section{URIC ACID AS A FUTURE BIOMARKER}

Uric acid represents the most important salivary antioxidant, in the circumstances of very low salivary thiols concentrations $(11,12)$. In an extracellular environment, like saliva, urate can scavenge hydroxyl radical, singlet oxygen, and peroxynitrite, especially when combined with ascorbic acid $(13,14)$. Consequently, in constellation with salivary oxidative stress, inflammation, collagen degradation biomarkers, uric acid plays an important role in oral pathology and may be regarded as a very useful future salivary biomarker.

\section{CONCLUSION}

Uric acid still remains a mysterious metabolite, but it promises to become a precious biomarker in order to assess the systemic and oral oxidative stress level.

\section{Acknowledgement}

In this article, all the authors have equal contributions.

7. Kang DH, Ha SK. Uric acid puzzle: Dual role as anti-oxidantand pro-oxidant. Electrolyte Blood Press. 2014, 12, 1-6.

8. Doherty M. New insights into the epidemiology of gout. Rheumatology (Oxford). 2009;48.

9. Feig DI, Kang DH, Johnson RJ. Uric acid and cardiovascular risk. N Engl J Med. 2008;359:1811-21.

10. Alvarez-Lario B, Macarron-Vicente $\mathrm{J}$. Is there anything good in uric acid? QJM. 2011;104:1015-24.

11. Totan A, Miricescu D, Parlatescu I, Mohora M, Greabu M. Possible salivary and serum biomarkers for oral lichen planus. Biotech Histochem. 2015, 90, 7, 552-8.

12. Stanescu II, Totan A, Rus F, Miricescu D, Mocanu B, Calenic B, Greabu M. Salivary Diagnosis - Clinical Uses in Assessing Oral Inflammation. Revista de chimie, 2017, 68(6), 1201-4

13. Battino M, Greabu M, Totan A et al. Oxidative stress markers in oral lichen planus. Biofactors 2008, 33, 301-10.

14. Sautin YY, Nakagawa T, Zharikov S, Johnson RJ. Adverse effects of the classic antioxidant uric acid in adipocytes: NADPH oxidase-mediated oxidative/nitrosative stress. Am J Physiol Cell Physiol 2009, 293, C584-C596 